\title{
Importance de différents paramètres climatiques sur la croissance et la formation du bois de pin rouge (Pinus resinosa Ait) en Ontario (Canada)
}

\author{
GR Larocque* \\ Ressources naturelles Canada, Service canadien des forêts, centre de foresterie des Laurentides, \\ 1055, rue du PEPS, case postale 3800, Sainte-Foy, PQ G1V 4C7, Canada
}

(Reçu le 21 mars 1995 ; accepté le 2 avril 1996)

\begin{abstract}
Summary - Importance of different climatic parameters on growth and wood formation of red pine (Pinus resinosa Ait) in Ontario (Canada). This study examines whether different monthly climatic parameters based on temperature and precipitation significantly influences radial growth and wood relative density of red pine (Pinus resinosa Ait). Cores were sampled on trees originating from a plantation established at $4.3 \times 4.3 \mathrm{~m}$ in Chalk River, Ontario, and were scanned on a X-ray densitometer. The following were measured on each ring: width and relative density relative densities of earlywood and latewood zones, minimal and maximal relative densities and latewood proportion. Significant relationships were derived for every dependent variable. Except for latewood relative density and maximum relative density, the independent variables that were significant consisted of previous and current year climatic variables based on mean temperatures, total precipitations, degree-days, number of days with precipitations and maximum number of continuous days without precipitation. These results indicate that wood formation of red pine is influenced by temperature as well as by the quantity and timing of precipitation.
\end{abstract}

ring width / radial growth / relative density / temperature / precipitation

Résumé - On examine dans cette étude si différents paramètres climatiques mensuels fondés sur les températures et les précipitations exercent une influence significative sur la croissance radiale et la densité relative du bois de pin rouge (Pinus resinosa Ait). Les arbres étudiés proviennent d'un peuplement artificiel établi initialement à $4,3 \times 4,3$ m à Chalk River, en Ontario. Les carottes de sondage échantillonnées ont été analysées avec un densitomètre aux rayons $\mathrm{X}$ pour obtenir les mesures de largeur et de densité relative de chaque cerne annuel, les densités relatives des zones de bois initial et final, les densités relatives minimales et maximales et la proportion du bois final. Des relations significatives ont été mises en évidence pour toutes les variables dépendantes. À l'exception de la densité relative de la zone de bois final et de la densité relative maximale, qui ne font intervenir que les paramètres de l'année courante, les régresseurs qui interviennent significativement consistent en des paramètres climatiques des années courantes et précédentes (températures moyennes, précipitations totales, degrés-jours, nombre de journées de précipitation et nombre maximum de journées continues sans précipitation). Ces résultats indiquent que la formation du bois de pin rouge est influencée aussi bien par les miveaux de température atteints et les quantités de précipitation que par leur répartition temporelle.

cerne / croissance radiale / densité relative / température / précipitation

*Tél : (418) 6485791 ; fax : (418) 6485849 ; courriel : glarocque @ cfl.forestry.ca 


\section{INTRODUCTION}

La croissance et la formation du matériel ligneux de tout arbre sont influencées par différents facteurs biotiques et abiotiques. Parmi les facteurs abiotiques, le climat joue un rôle prépondérant (Jordan et Lockaby, 1990). De nombreuses études, qui ont examiné spécifiquement l'effet des fluctuations climatiques sur la croissance, l'ont bien démontré (par exemple, Perala, 1983 ; Wijk, 1986 ; Ford et al, 1987a, b ; Le Goff et Ottorini, 1993 ; Santini et al, 1994 ; Vose et Swank, 1994 ; Yin et al, 1994). Au cours des dernières décennies, la reconstitution de séries chronologiques de paramètres climatiques à partir de séries dendrochronologiques a pris de plus en plus d'ampleur (Cook et al, 1987 ; D'Arrigo et al, 1992). Le paramètre de croissance le plus utilisé a été la largeur des cernes annuels. Normalement, les données d'épaisseurs de cernes individuels obtenues d'arbres âgés sont standardisées afin de tempérer les variations reliées à l'âge et à la compétition. Des relations sont ensuite établies entre les séries standardisées et les séries synchrones de paramètres climatiques disponibles. De bons exemples récents sont les travaux de Jones et al (1991), Eriksson et al (1992), Archambault et Bergeron (1992), Cutler et al (1993), Foster et Leblanc (1993), Becker et al (1994) et Tessier et al (1994).

Plus récemment, on a utilisé les méthodes de la dendrochronologie pour évaluer l'impact de la pollution et du changement climatique d'origine anthropique sur la croissance (par exemple, Becker, 1987 ; Bert et Becker, 1990 ; Van Deusen, 1990 ; Graumlich, 1993 ; Jones et al, 1993 ; Leblanc, 1993 ; Reams et al, 1993 ; Power, 1994). Si ces changements se produisent à un rythme accéléré, les impacts sociaux, environnementaux et économiques risquent d'être importants. Par conséquent, une bonne connaissance des relations entre les facteurs climatiques et la croissance est nécessaire afin de faciliter l'évaluation de l'impact de ces changements globaux sur les approvisionnements en matière ligneuse (Robertson et al, 1990 ; Eriksson et al, 1992).

La majorité des études de ce type sont basées sur l'établissement de relations statistiques qui prédisent la croissance en diamètre ou en hau- teur à partir de paramètres climatiques. On peut s'attendre à ce que la densité du bois soit aussi affectée significativement par le climat environnant puisque, comme la croissance en diamètre, elle est tributaire de l'allocation en carbone, de l'activité hormonale à l'intérieur de l'arbre (Denne, 1979) et du taux de division cellulaire au niveau du cambium. Toutes ces fonctions physiologiques dépendent largement des conditions climatiques (Kozlowski et al, 1991 ; Smith 1985). Cependant, si l'on considère que la densité du bois reflète non seulement le rythme de production des cellules et leurs dimensions mais aussi l'épaisseur de leurs parois, tandis que la largeur du cerne reflète seulement les dimensions et le rythme de production des cellules, ces deux caractéristiques ne devraient pas nécessairement dépendre des mêmes facteurs climatiques. Par exemple, Conkey (1988) et D'Arrigo et al (1992) mentionnent que la densité maximale du cerne annuel est plus sensible au stress environnemental que la largeur du cerne. Kienast et al (1987) suggèrent, pour des sites alpins, qu'il est préférable d'utiliser la densité maximale du bois pour reconstruire les variations de températures et la largeur des cernes annuels pour la reconstruction des variations de précipitations. Toutefois, D'Arrigo et al (1992) spécifient que des études plus exhaustives devraient prendre en compte d'autres caractéristiques du cerne comme la densité des zones de bois initial et final.

L'impact des fluctuations climatiques sur le développement de la fibre ligneuse a été étudié par Parker et Henoch (1971), Hughes et al (1984), Kienast et al (1987), Conkey (1988), Cregg et al (1988), Robertson et Jozsa (1988), Wilkes (1989), Robertson et al (1990) et D'Arrigo et al (1992). Cependant, à l'exception des études de Robertson et Jozsa (1988) et de Robertson et al (1990) sur le sapin de Douglas (Pseudotsuga menziesii (Mirb) Franco), l'effet du régime climatique sur la densité du bois n'a été que partiellement examiné jusqu'à maintenant. En effet, les études existantes se caractérisent par un nombre restreint de paramètres climatiques analysés (par exemple, Conkey, 1988 ; Hughes et al, 1984), par l'utilisation de la densité maximale seulement (par exemple, 
Tableau I. Statistiques de base des arbres retenus.

\begin{tabular}{lccccc} 
& \multicolumn{2}{c}{ dhp $\overline{(\mathrm{cm})}$} & & \multicolumn{2}{c}{ Hauteur $(\mathrm{m})$} \\
\cline { 2 - 3 } \cline { 5 - 6 } Âge & Moyenne & Écart type & & Moyenne & Écart type \\
\hline 13 & 4,26 & 2,57 & & 2,91 & 0,88 \\
33 & 28,37 & 3,92 & 14,62 & 1,39 \\
\hline
\end{tabular}

Conkey, 1988 ; Hughes et al, 1984 ; Kienast et al, 1987), ou par l'absence de fonctions de réponse (par exemple, Parker et Henoch, 1971 ; Kienast et al, 1987 ; Wilkes, 1989 ; D’Arrigo et al, 1992). Dans la présente étude pour le pin rouge (Pinus resinosa Ait), il sera examiné jusqu'à quel point les différentes caractéristiques du cerne telles que la largeur et les densités relatives des zones de bois initial et final réagissent aux fluctuations climatiques interannuelles.

\section{SITE, MATÉRIEL ET MÉTHODE}

Les arbres étudiés proviennent de plantations de pins rouges à espacements variables localisées sur la propriété d'Énergie atomique du Canada Limitée à Chalk River, en Ontario (Canada). Les plantations ont été établies en 1953 avec des plants âgés de quatre ans à des espacements initiaux variant entre 1,2 et $4,3 \mathrm{~m}$ sur des cultures abandonnées dont le sol se compose de sable fin à moyen. L'indice de fertilité a été estimé à $24,4 \mathrm{~m}$ à 50 ans par Stiell et Berry $(1973,1977)$, ce qui indique une très bonne productivité, puisque la valeur maximale estimée par Beckwith et al (1983) pour le pin rouge dans le sud de l'Ontario est de $26 \mathrm{~m}$. Le pin rouge est d'ailleurs communément planté sur ce type de station dans l'est du Canada.

Quand l'essai d'espacement fut établi, tous les arbres furent identifiés et numérotés sur le terrain. Le dhp (diamètre à hauteur de poitrine) a été mesuré sur tous les arbres à 13, 18, 23, 28 et 33 ans. À l'origine, des carottes de sondage furent échantillonnées sur 30 arbres dans chaque classe d'espacement (une carotte par arbre) afin d'étudier l'effet de différents espacements initiaux sur la densité relative du bois, c'est-àdire le rapport entre la densité de la fibre ligneuse et la densité de l'eau. Cette partie de l'étude est achevée (Larocque et Marshall,
1995). Pour atteindre les objectifs de la présente étude, seuls les arbres de l'espacement de 4,3 m ont été retenus afin de minimiser les effets de la compétition (tableau I), ce qui est normalement suggéré pour ce type d'étude (Fritts, 1976). Les carottes ont été débitées en lames d'épaisseur de $1,57 \mathrm{~mm}$ avec un appareil pneumatique à deux scies circulaires. Elles ont ensuite été traitées avec une solution de benzène et d'alcool $(2: 1)$ pour en extraire les composés chimiques comme les gommes et les résines. Les données de densité relative ont été obtenues avec un densitomètre aux rayons $\mathrm{X}$ situé au laboratoire de Forintek Canada Corp. à Vancouver, en Colombie-Britannique. Les mesures suivantes ont été obtenues pour chaque cerne annuel : largeur et densité relative, largeurs et densités relatives des zones de bois initial et final, et densités relatives minimales et maximales (fig 1). De plus amples renseignements sur les procédés sont contenus dans Larocque et Marshall (1995).

Pour chacune des sept variables dépendantes, la première étape d'analyse a consisté à standardiser les données en appliquant la méthodologie suggérée par Fritts (1976) et Van Deusen et Reams (1993) afin de tempérer l'effet de l'âge et de la compétition : i) dérivation d'une équation polynomiale d'ordre deux, trois ou quatre pour chaque série chronologique ;ii) calcul d'un indice de standardisation fondé sur la division des observations par les prédictions fournies par l'équation ; et iii) calcul de la moyenne des séries d'indices.

Les données climatiques ont été fournies par la station météorologique d'Énergie atomique du Canada Limitée située à environ $3 \mathrm{~km}$ du site expérimental à Chalk River (Jay et Wildsmith, 1991). Seules les données couvrant la saison de croissance, soit d'avril à septembre, ont été utilisées. Pour chaque mois, les huit variables 

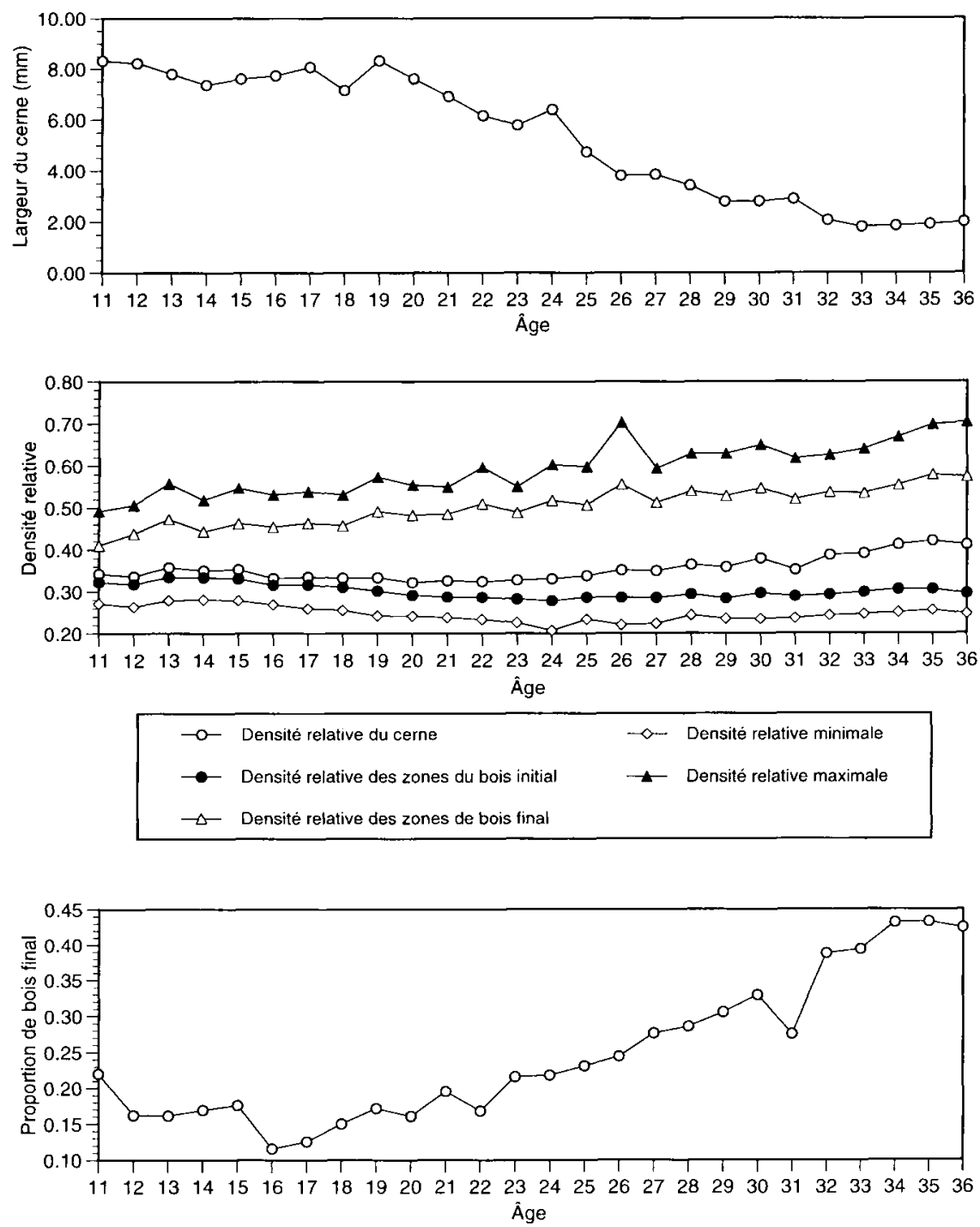

Fig 1. Moyennes des largeurs du cerne, des densités relatives et des proportions de bois final pour l'ensemble des pins rouges retenus.

suivantes ont été calculées à partir des observations journalières : température moyenne des minimums journaliers (MINT), température moyenne (MOYT), température moyenne des maximums journaliers (MAXT), précipitations totales (PREC), nombre de degrés-jours au des- sus de $10^{\circ} \mathrm{C}$ (DD10) et de $20^{\circ} \mathrm{C}$ (DD20), nombre de journées pluvieuses (RAD) et nombre maximal de journées continuelles sans pluie (valeur maximale des différentes périodes de journées continuelles sans pluie) (DRD) (tableau II, fig 2). 
Tableau II. Sommaire et abréviations des variables climatiques étudiées pour les mois d'avril à septembre.

\begin{tabular}{ll}
\hline Abréviation & Description \\
\hline MINTAVR...SEP & Température mensuelle moyenne des minimums journaliers $\left({ }^{\circ} \mathrm{C}\right)$ \\
MOYTAVR...SEP & Température mensuelle moyenne $\left({ }^{\circ} \mathrm{C}\right)$ \\
MAXTAVR...SEP & Température mensuelle moyenne des maximums journaliers $\left({ }^{\circ} \mathrm{C}\right)$ \\
DD10AVR...SEP & Degrés-jours mensuels au dessus de $10^{\circ} \mathrm{C}$ \\
DD20AVR...SEP & Degrés-jours mensuels au dessus de $20^{\circ} \mathrm{C}$ \\
PRECAVR...SEP & Précipitations mensuelles (mm) \\
RADAVR...SEP & Nombre mensuel de journées de précipitations (jours) \\
DRDAVR...SEP & Nombre mensuel maximal de journées continuelles sans précipitations (jours) \\
SNOAVR...SEP & Précipitations mensuelles (neige, mm) \\
\hline
\end{tabular}

a Chaque nom de variable est formé d'un terme correspondant à un mois donné (par exemple, MINTAVR, MINTMAI, MINTJUN, MINTJUL, MINTAOU, MINTSEP pour les températures mensuelles moyennes des minimums journaliers d'avril, mai, juin, juillet, août et septembre, respectivement).

L'analyse des données a été exécutée par la méthode de régression progressive pas à pas avec le logiciel Sas (Sas Institute Inc, 1989). Cette technique est considérée comme optimale lorsque beaucoup de variables sont à analyser (Draper et Smith, 1981). Comme les conditions climatiques d'une saison de croissance particulière affectent la croissance et la formation du bois à la saison suivante pour de nombreuses espèces (Zimmermann et Brown, 1971 ; Fritts, 1976, 1982 ; Jacoby et D'Arrigo, 1989 ; Kozlowski et al, 1991), les analyses de régression ont été effectuées de sorte que les variables climatiques de l'année courante qui s'achève en septembre et de l'année précédente soient incluses comme variables explicatives.

\section{RÉSULTATS ET DISCUSSION}

Les analyses de régression à partir des variables climatiques ont fourni des équations contenant de deux à six régresseurs (figs 3 et 4 , tableau III). Tous les paramètres retenus interviennent significativement au niveau de probabilité de 0,05 et des coefficients de détermination relativement élevés ont été obtenus, particulièrement pour la largeur du cerne, la densité relative de la zone de bois initial, la densité relative minimale et la proportion du bois final. La plus faible valeur du coefficient de détermination obtenue pour la densité relative du cerne annuel par rapport à la valeur obtenue pour la densité relative de la zone de bois initial s'explique probablement par le fait que la densité relative du cerne reflète à la fois les densités relatives de deux zones de bois qui sont affectées par des facteurs climatiques indépendants. Les équations de régression calculées ne posent pas de problèmes majeurs d'autocorrélation élevée des résidus (tableau III).

Le nombre de régresseurs qui interviennent significativement dans les fonctions de réponse est faible par rapport au nombre de paramètres climatiques initialement retenus pour les analyses de régression. Quatre raisons peuvent être invoquées : i) fluctuations peu prononcées de certains paramètres climatiques, c'est le cas notamment des températures par rapport aux autres paramètres climatiques ; ii) comme les peuplements étudiés sont situés sur un type de station où le pin rouge se distingue par sa bonne productivité, on peut supposer que les conditions météorologiques générales ne causent pas de stress climatique important ; iii) l'effet des différents paramètres climatiques sur les processus physiologiques change au cours de la saison de croissance (Fritts, 1982) ; iv) les différents processus physiologiques ne sont pas affectés pareillement par les paramètres climatiques (Kozlowski et al, 1991). Par exemple, Larson $(1963,1964,1969)$ note que la formation du bois initial et du bois final dépend de l'équilibre complexe entre la synthèse d'hormones de croissance (auxines) et la disponibilité des produits de la photosynthèse.

À l'exception de la densité relative de la zone de bois final et de la densité relative maximale, les fonctions de réponse mettent en évidence des 

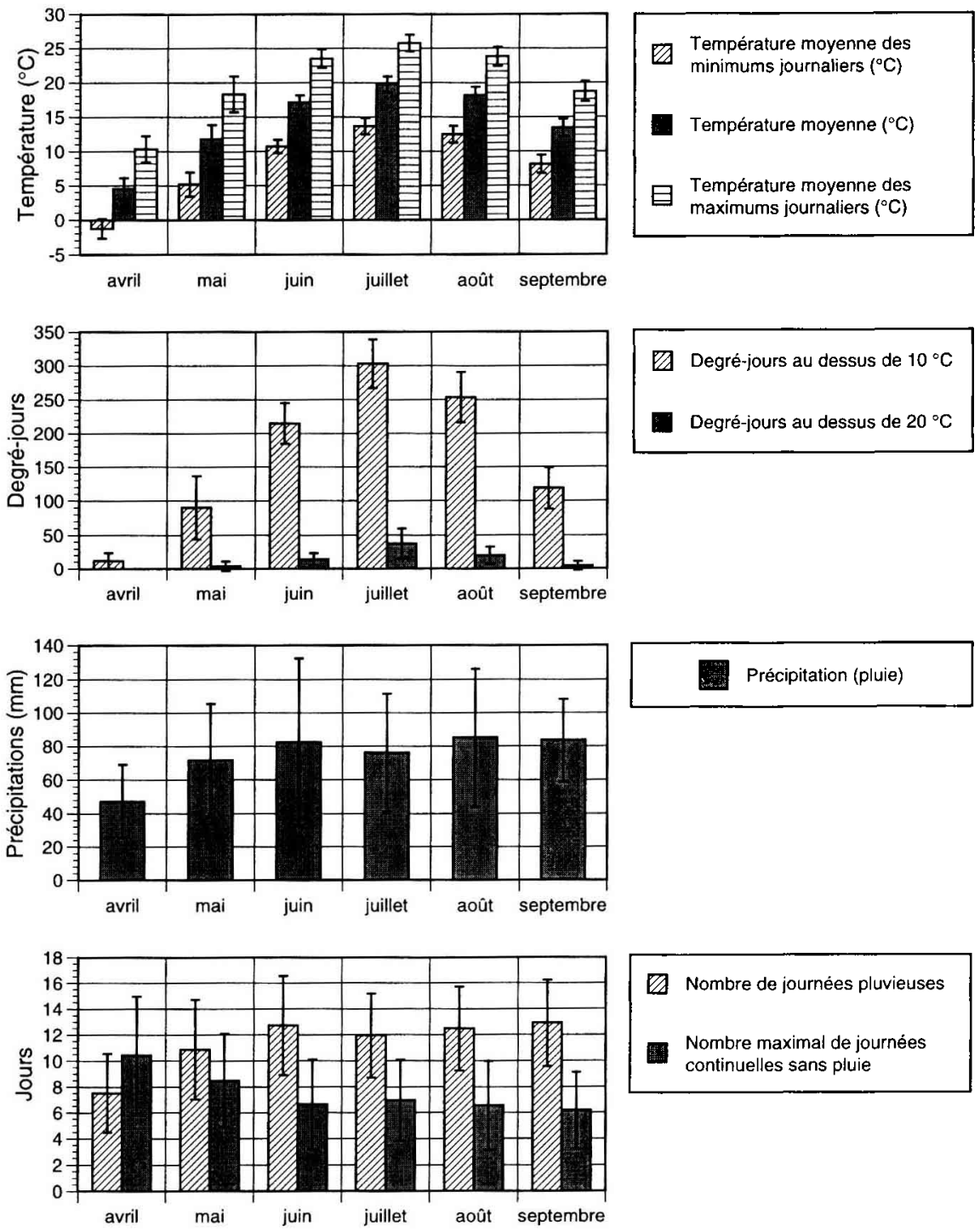

Nombre de journèes pluvieuses

Nombre maximal de journées continuelles sans pluie

Fig 2. Moyennes des températures, des degrés-jours, des précipitations, du nombre de journées pluvieuses el du nombre maximal de journées continues sans pluie entre avril et septembre, de 1963 à 1985, près du peuplement sur la propriété d'Énergie atomique du Canadà Limitée à Chalk River.

variables climatiques des saisons courantes et précédentes (figs 3 et 4 ). L'intervention significative de variables climatiques de l'année précédente reflète bien le fait que le pin rouge est une espèce à croissance déterminée (Duff et Nolan, 1958 ; Whitmore et Zahner, 1966 ; Zahner et Stage, 1966 ; Clements, 1970 ; Olofinboda et Kozlowski, 1973 ; Daniel et al, 1979) : les 
Tableau III. Statistiques des équations linéaires multiples dérivées.

\begin{tabular}{|c|c|c|c|c|}
\hline Variable dépendante & Test $F$ & $\begin{array}{c}\text { Écan type } \\
\text { des estimations }\end{array}$ & $\mathrm{R}^{2}$ & $\begin{array}{l}\text { Autocorrélation du premier } \\
\text { ordre sur les résidus }\end{array}$ \\
\hline Largeur du cerne & $13,24 * *$ & 0,042 & 0,80 & 0,003 \\
\hline Densité relative du cerne & $9,45 * *$ & 0,014 & 0,69 & $-0,17$ \\
\hline $\begin{array}{l}\text { Densité relative de la zone } \\
\text { de bois initial }\end{array}$ & $15,15^{* *}$ & 0,009 & 0,82 & $-0,19$ \\
\hline $\begin{array}{l}\text { Densité relative de la zone } \\
\text { de bois final }\end{array}$ & $14,07 * *$ & 0,016 & 0,70 & $-0,26$ \\
\hline Densité relative minimale & $16,17^{* *}$ & 0,016 & 0,79 & 0,05 \\
\hline Densité relative maximale & $8,40^{* *}$ & 0,034 & 0,47 & $-0,07$ \\
\hline Proportion de bois final & $17,04 * *$ & 0,046 & 0,87 & $-0,22$ \\
\hline
\end{tabular}

** Significatif au seuil de probabilité de $0,01(\alpha<0,01)$.

conditions climatiques au moment de l'aoûtement ont une influence sur la croissance et la formation du bois au cours de la saison de croissance suivante.

\section{Année courante}

Pour les températures, seules la température moyenne d'avril et la température moyenne des minimums journaliers de juillet sont incluses dans les fonctions de réponse portant respectivement sur la largeur du cerne et la proportion du bois final (figs 3 et 4 ). Les nombres de degrés-jours au dessus de 10 et $20^{\circ} \mathrm{C}$ sont reliés significativement aux variables dépendantes plus souvent que les températures : largeur du cerne, densité relative du cerne, densité relative de la zone de bois final, densité relative minimale et proportion de bois final. Ceci suggère que la durée de la période pendant laquelle un certain niveau de température se maintient a autant d'importance pour la croissance et la formation du bois que le niveau de température atteint. Toutefois, pour la largeur du cerne et la proportion de bois final, on a obtenu simultanément des relations significatives avec les degrés-jours et les températures.

En général, les variables climatiques portant sur les précipitations ont une importance relative moins élevée que les températures et degrés-jours dans la prédiction de la largeur du cerne et des densités relatives. Ceci peut s'expliquer par le fait que le site étudié est caractérisé par un bon substrat pour le pin rouge de telle sorte qu'il n'y a pas de déficit hydrique important.
En accord avec ce qui a été mentionné par Creber et Chaloner (1984), nos résultats indiquent que les précipitations cumulées ne constituent pas le facteur le plus limitant, mais que leur répartition au cours de la saison est aussi essentielle puisque le nombre de journées de précipitations et le nombre mensuel maximal de journées continuelles sans précipitations constituent des régresseurs significatifs plus souvent que les quantités de précipitations. L'effet négatif du nombre de journées de précipitations en juillet sur la densité relative du cerne est combiné à l'effet positif du nombre maximum de journées continuelles sans précipitations en juillet sur la densité relative de la zone de bois initial. Ces résultats sont compatibles avec les études de Zahner et al (1964), Whitmore et Zahner (1966), Larson $(1964,1969)$ et Zimmermann et Brown (1971) qui ont observé que la transition du bois initial au bois final est favorisée par de bas niveaux d'humidité.

\section{Année précédente}

L'effet des températures de l'année précédente sur la croissance et la formation du bois se limite aux températures moyennes d'août et de septembre (figs 3 et 4). Leur intervention négative sur la largeur du cerne et la densité relative de la zone de bois initial suggère que des températures trop élevées en août et en septembre ont un impact négatif sur la formation du bois au cours de l'année suivante. Des températures élevées pendant la nuit lors de la formation des bourgeons peuvent expliquer ces résultats. 

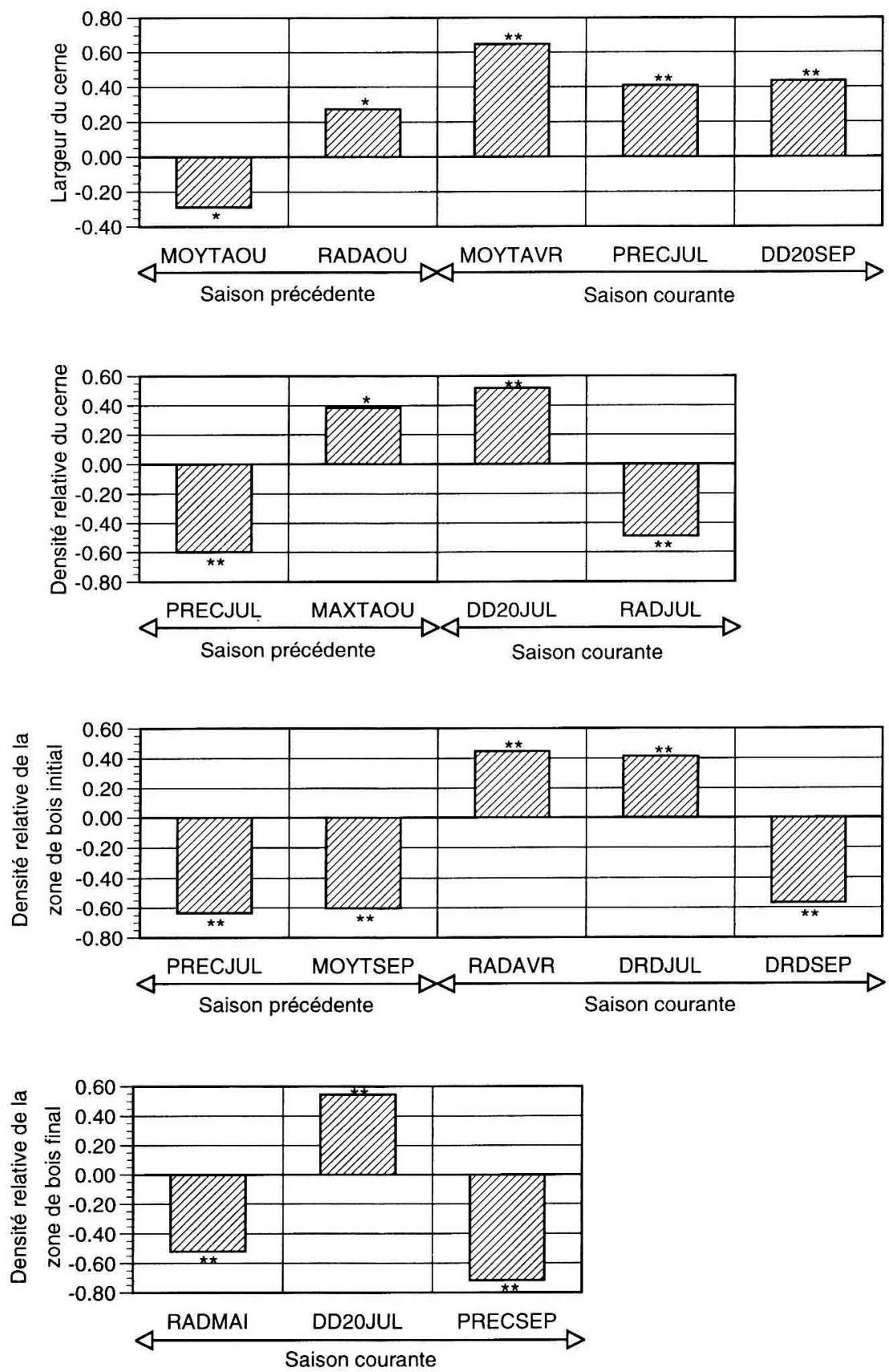

Fig 3. Coefficients de régression standardisés pour la largeur du cerne annuel et les densités relatives du cerne annuel et des zones de bois initial et final. ${ }^{*}$ Significatif au seuil de probabilité de 0,$05 ;{ }^{*}$ significatif au seuil de probabilité de 0,01 . 

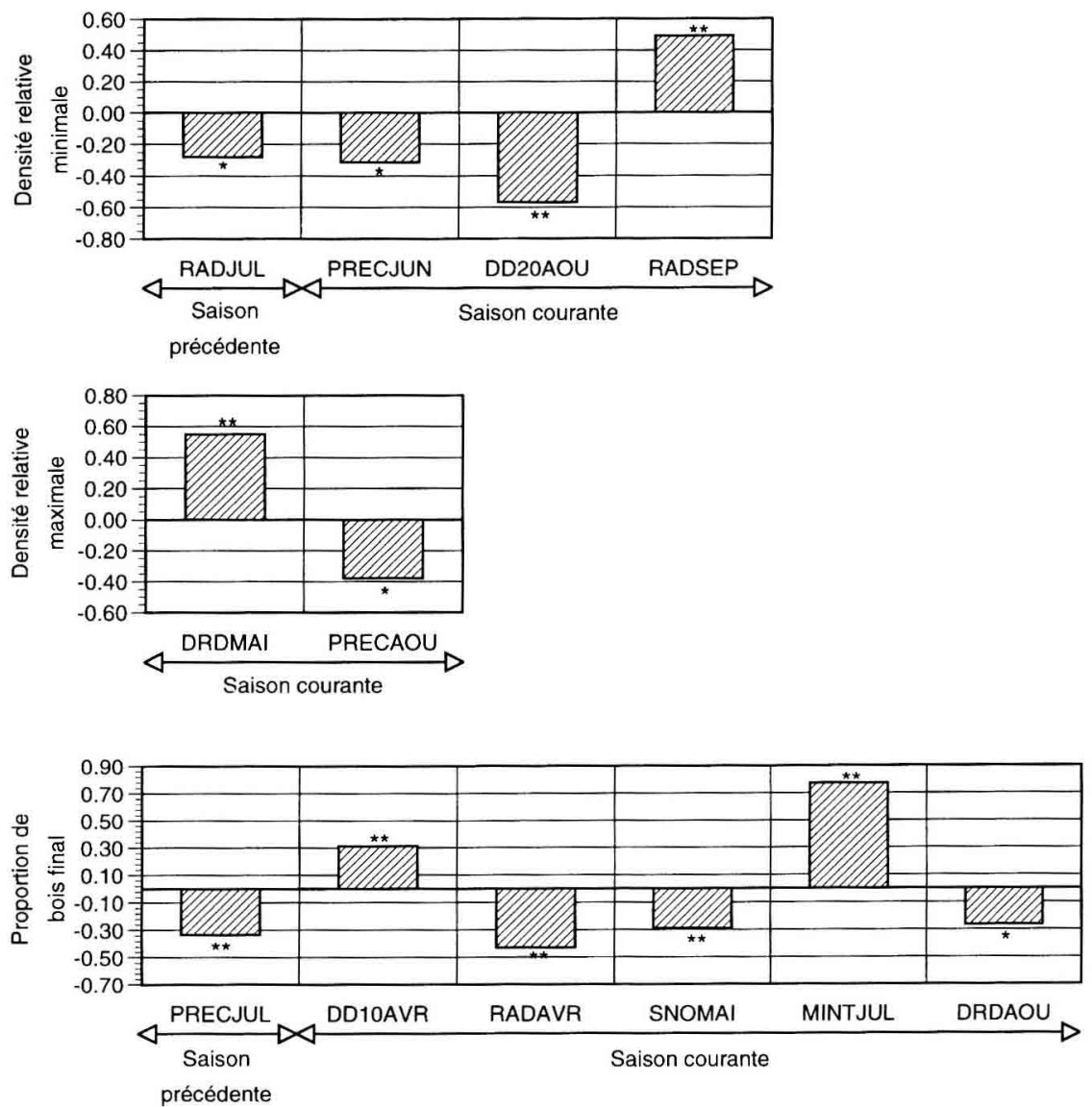

Fig 4. Coefficients de régression standardisés pour les densités relatives minimales et maximales et la proportion de bois final. *Significatif au seuil de probabilité de 0,$05 ;{ }^{* *}$ significatif au seuil de probabilité de 0,01 .

Même si aucune preuve directe n'a été trouvée pour le pin rouge dans la littérature, les études de Deal et al (1990) sur l'épinette du Colorado (Picea pungens Engelm) et d'Odlum et Colombo (1989) sur l'épinette noire (Picea mariana (Mill) BSP), qui sont deux espèces à croissance déterminée, montrent que des températures nocturnes élevées lors de la formation des bourgeons pouvaient avoir un impact négatif sur leur développement et leur débourrement au début de la saison de croissance de l'année suivante et ultérieurement sur la croissance des pousses annuelles. Cook et al (1987) ont aussi obtenu des relations négatives entre les températures de juillet et d'août de l'année précédente et la croissance radiale de l'épinette rouge ( Picea rubens Sarg). Deal et al (1990) ont suggéré que des températures nocturnes élevées accroissent le taux de respiration, augmentant par le fait même la perte d'hydrates de carbone.

Les relations négatives entre les quantités de précipitation en juillet de l'année précédente et la densité relative du cerne, la densité relative de la zone de bois initial et la proportion de bois 
final, de même que la relation négative entre le nombre de journées de précipitations du même mois et la densité relative minimale suggèrent que les précipitations, en favorisant la croissance radiale et la synthèse des auxines pour l'année courante (Fritts, 1976 ; Daniel et al, 1979 ; Kozlowski et al, 1991), retardent la formation des bourgeons, ce qui a probablement un effet négatif sur la formation du bois au début de la saison de croissance suivante.

\section{Comparaison avec d'autres études}

L'équation obtenue pour la largeur du cerne annuel est caractérisée par un degré d'explication (mesuré par les coefficients de corrélation ou de détermination) égal ou supérieur à ceux fournis par d'autres études (par exemple, Cook et al, 1987 ; Kim et Siccama, 1986 ; Cregg et al, 1988 ; Eriksson et al, 1992 ; Jordan et Lockaby, 1990 ; Robertson et al, 1990 ; Yin et al, 1994). Comme il a été mentionné précédemment, peu d'études ont établi des relations entre des paramètres climatiques et la densité relative du bois. Pour la densité relative des zones de bois initial, on peut comparer avec les travaux de Robertson et al (1990) pour le sapin de Douglas en Colombie-Britannique. De prime abord, le degré d'explication obtenu dans la présente étude apparaît supérieur à celui obtenu dans les relations établies par Robertson et al (1990). Ils ont obtenu des coefficients de détermination entre 0,43 et 0,69 pour des équations qui mettent en évidence des relations significatives avec la température moyenne de la saison de croissance et le déficit en eau au printemps. Toutefois, il est possible que leurs équations aient été relativement moins explicatives du fait qu'elles étaient basées sur des moyennes bimensuelles. Pour la densité relative des zones de bois final, Robertson et al (1990) ont obtenu des coefficients de détermination entre 0,38 et 0,57 pour des équations basées sur la température moyenne de la saison de croissance et le déficit en eau de l'été. L'équation obtenue dans la présente étude est plus explicative, probablement pour la même raison que celle discutée plus haut à propos de la densité relative des zones de bois initial.

La qualité de l'ajustement fourni par l'équation calculée pour la densité relative maximale est médiocre en comparaison des corrélations obtenues par Hughes et al (1984) $(0,63$ à 0,76$)$ et Parker et Henoch (1971) $(0,79$ à 0,85), mais se compare favorablement à la relation obtenue par Conkey (1988) $(0,60)$. Le fait que la densité maximale reflète une phase de la construction du cerne qui se produit en une période très courte durant la saison de croissance explique probablement cette faible qualité de l'ajustement. En revanche, l'ajustement obtenu pour la proportion du bois final dans la présente étude est meilleur que celui obtenu par Robertson et al (1990). L'ajustement médiocre obtenu pour la densité relative du cerne annuel par rapport à ceux obtenus pour les densités relatives des zones de bois initial et final s'explique pro- bablement par le fait que la densité relative du cerne reflète à la fois les densités relatives des deux zones de bois qui sont affectées par des facteurs climatiques différents.

\section{CONCLUSION}

Les résultats de cette étude démontrent bien que l'influence des différents paramètres climatiques sur la croissance radiale et le développement de la fibre ligneuse résulte d'un équilibre complexe : certains paramètres climatiques ont une influence positive sur la croissance, mais une influence négative sur la densité relative du bois. Le degré élevé d'explication des fonctions de réponse obtenue tant pour la largeur des cernes annuels que pour la densité relative des zones de bois initial et final suggère que ce genre de modèle peut être utile pour l'évaluation de l'impact du changement climatique sur la production de la fibre ligneuse. Les résultats de la présente étude indiquent que l'analyse des indices basés sur des paramètres autres que la largeur du cerne peut fournir une évaluation plus détaillée des effets des fluctuations climatiques sur la formation de la fibre ligneuse. La suite logique de la présente étude serait d'analyser des peuplements semblables situés dans des conditions climatiques et écologiques extrêmement différentes.

\section{REMERCIEMENTS}

Je tiens à exprimer mes plus sincères remerciements à $\mathrm{P}$ Davis, du laboratoire d'Énergie atomique du Canada Limitée à Chalk River, qui a aimablement mis à ma disposition les données 
climatiques pour les fins de la présente étude, au $\mathrm{D}^{r} \mathrm{RM}$ Kellogg, chercheur retraité de Forintek Canada Corp, pour ses conseils extrêmement utiles lors de la planification de la présente étude, à LA Jozsa, de Forintek Canada Corp, et à J Richards et S Johnson, anciennement de Forintek Canada Corp, pour leur précieuse collaboration lors de l'utilisation du densitomètre aux rayons $\mathrm{X}$. Mes remerciements s'adressent aussi au $D^{\text {rs }} S$ Magnussen et WM Strome et à $\mathrm{P}$ Boross, de l'Institut forestier national de Petawawa, pour leurs suggestions lors de la révision du manuscrit.

\section{RÉFÉRENCES}

Archambault S, Bergeron Y (1992) An 802-year treering chronology from the Quebec boreal forest. Can J For Res 22, 674-682

Becker M (1987) Bilan de santé actuel et rétrospectif du sapin (Abies alba Mill) dans les Vosges. Étude écologique et dendrochronologique. Ann Sci For $44,379-402$

Becker M, Nieminen TM, Gérémia F (1994) Shortterm variations and long-term changes in oak productivity in northeastern France. The role of climate and atmospheric $\mathrm{CO}_{2}$. Ann Sci For 51 , 477-492

Beckwith AF, Roebbelen P, Smith VG (1983) Red pine plantation growth and yield tables. Ontario Ministry of Natural Resources, Ontario Tree Improvement and Forest Biomass Institute, Forest Research Report No 108

Bert GD, Becker HM (1990) Vitalité actuelle et passée du sapin (Abies alba Mill) dans le Jura. Étude dendroécologique. Ann Sci For 47, 395-412

Clements JR (1970) Shoot responses of young red pine to watering applied over two seasons. Can J Bot $48,75-80$

Conkey LE (1988) Decline in old-growth red spruce in western Maine: an analysis of wood density and climate. Can J For Res 18, 1063-1068

Cook ER, Johnson AH, Blasing TJ (1987) Forest decline: modeling the effect of climate in tree rings. Tree Physiol 3, 27-40

Creber GT, Chaloner WG (1984) Influence of environmental factors on the wood structure of living and fossil trees. Bot Rev 50, 357-448

Cregg BM, Dougherty PM, Hennessey TC (1988) Growth and wood quality of young loblolly pine trees in relation to stand density and climatic factors. Can J For Res 18, 851-858
Cutler DF, Bridge MC, Gasson PE (1993) An introduction to dendrochronological work on windblown trees at Kew and Wakehurst Place. Forestry 66, 225-232

D'Arrigo RD, Jacoby GC, Free RM (1992) Tree-ring width and maximum latewood density at the North American tree line: parameters of climatic change. Can J For Res 22, 1290- 1296

Daniel TW, Helms JA, Baker FS (1979) Principles of Silviculture, 2nd ed. McGraw-Hill, New York, NY, États-Unis

Deal DL, Raulston JC, Hinesley LE (1990) High temperature effects on apical bud morphology, dark respiration, and fixed growth of blue spruce. Can J For Res 12, 1871-1877

Denne MP (1979) Wood structure and production within the trunk and branches of Picea sitchensis in relation to canopy formation. Can J For Res 9 , $406-427$

Draper NR, Smith H (1981) Applied Regression Analysis, 2nd ed. John Wiley \& Sons, New York, NY, États-Unis

Duff G.H, Nolan NJ (1958) Growth and morphogenesis in the Canadian forest species. III. The time scale of morphogenesis at the stem apex of Pinus resinosa Ait. Can J Bot 36, 687-707

Eriksson M, Lin, C-D, Thomas CE, Lohrey RE (1992) Dendroecological modelling of thinned and unthinned longleaf pine stands in the western gulf region. In : Proceedings of the Seventh Biennial Southern Silvicultural Research Conference, Mobile, Alabama, 17-19 November 1992 (JC Brisette, ed), USDA Forest Service, Southern Forest Experiment Station, General Technical Report SO-93, 585-592

Ford ED, Milne R, Deans JD (1987a) Shoot extension in Picea sitchensis. I. Seasonal variation within a forest canopy. Ann Bot 60, 531-542

Ford ED, Milne R, Deans JD (1987b) Shoot extension in Picea sitchensis. II. Analysis of weather influences on daily growth rate. Ann Bot 60, 543-552

Foster JR, Leblanc DC (1993) A physiological approach to dendroclimatic modeling of oak radial growth in the midwestern United States. Can J For Res 23, 783-798

Fritts HC (1976) Tree Rings and Climate. Academic Press, New York, NY, États-Unis

Fritts HC (1982) The climate-growth response. In : Climate from Tree Rings (MK Hughes, PM Kelly, JR Pilcher, VC Lamarche Jr, eds), Cambridge University Press, Cambridge, MA, États-Unis, 33-38

Graumlich LJ (1993) Response of tree growth to climatic variation in the mixed conifer and 
deciduous forests of the upper Great Lakes region. Can J For Res 23, 133-143

Hughes MK, Schweingruber FH, Cartwright D, Kelly PM (1984) July-August temperature at Edinburgh between 1721 and 1975 from tree-ring density and width data. Nature 308, 341-344

Jacoby GC Jr., D'Arrigo R (1989), Reconstructed northern hemisphere annual temperature since 1671 based on high-latitude tree-ring data from North America. Climatic Change 14, 39-59

Jay PC, Wildsmith DP (1991) Climatological data at Chalk River Laboratories, 1963-1990. Atomic Energy of Canada Limited, Environmental Research Branch, Chalk River Laboratories, TR-515

Jones EA, Reed DD, Cattelino PJ, Mroz GD (1991) Seasonal shoot growth of planted red pine predicted from air temperature degree-days and soil water potential. For Ecol Manage 46, 201-214

Jones EA, Reed DD, Mroz GD, Liechty HO, Cattelino PJ (1993) Climate stress as a precursor to forest decline: paper birch in northern Michigan. Can J For Res 23, 229-233

Jordan, DN, Lockaby BG (1990) Time series modelling of relationships between climate and longterm radial growth of loblolly pine. Can J For Res 20, 738-742

Kienast F, Schweingruber FH, Bräker OU, Schär E (1987) Tree-ring studies on conifers along ecological gradients and the potential of single-year analyses. Can J For Res 17, 683-696

Kim E, Siccama TG (1986) The influence of temperature and soil moisture on the radial growth of northern hardwood tree species at Hubbard Brook Experimental Forest, New Hampshire, USA. In : Proceedings of the International Symposium on Ecological Aspects of Tree-ring Analysis, 17-21 August 1986, Tarrytown, NY (GC Jacoby Jr, JW Hornbeck, eds), US Department of Energy, Office of Energy Research, Washington, DC, États-Unis, 26-37

Kozlowski TT, Kramer PJ, Pallardy SG (1991) The Physiological Ecology of Woody Plants. Academic Press, New York, NY, États-Unis, 657 p

Larocque GR, Marshall PL (1995) Wood relative density development in red pine ( $P$ inus resinosa Ait) stands as affected by different initial spacings. For Sci 41, 709-728

Larson PR (1963) The indirect effect of drought on tracheid diameter in red pine. For Sci 9, 52-62

Larson PR (1964) Some indirect effects of environment on wood formation. In : The Formation of Wood in Forest Trees (MH Zimmermann, ed), Academic Press, New York, NY, États-Unis, 345-365
Larson PR (1969) Wood formation and the concept of wood quality. Yale University, School of Forestry, New Haven, CT, États-Unis, Bulletin No 74, 54 p

Leblane DC (1993) Temporal and spatial variation of oak growth-climate relationships along a pollution gradient in the midwestern United States. Can J For Res 23, 772-782

Le Goff N, Ottorini JM (1993) Thinning and climate effects on growth of beech (Fagus sylvatica L) in experimental stands. For Ecol Manage 62, 1-14

Odlum KD, Colombo SJ (1989) The influence of night temperature under declining photoperiod on bud initiation in black spruce seedlings. Can J For Res 19, 274-275

Olofinboda MO, Kozlowski TT (1973) Accumulation and utilisation of carbohydrate reserves in shoot growth of Pinus resinosa. Can J For Res 3, 346-353

Parker ML, Henoch WES (1971) The use of Engelmann spruce latewood density for dendrochronological purposes. Can J For Res 1, 90-98

Perala DA (1983) Modeling aspen and red pine shoot growth to daily weather variations. USDA Forest Service, North Central Forest Experiment Station, Research Paper NC-236

Power SA (1994) Temporal trends in twig growth of Fagus sylvatica $\mathrm{L}$ and their relationships with environmental factors. Forestry 67, 13-30

Reams GA, Nicholas NS, Zedaker SM (1993) Two hundred year variation of southern red spruce radial growth as estimated by spectral analysis. $\mathrm{Can}$ $J$ For Res 23, 291-301

Robertson EO, Jozsa LA (1988) Climatic reconstruction from tree rings at Banff. Can J For Res 18, $888-900$

Robertson EO, Jozsa LA, Spittlehouse DL (1990) Estimating Douglas-fir wood production from soil and climate data. Can J For Res 20, 357-364

Santini A, Bottacci A, Gellini R (1994) Preliminary dendroecological survey on pedunculate oak (Quercus robur L) stands in Tuscani (Italy). Ann Sci For 51, 1-10

Sas Institute Inc (1989) SAS/STAT ${ }^{\circledR}$ user's guide, Version 6, Fourth Edition, Volume 2, SAS Institute Inc, Cary, NC, États-Unis, $846 \mathrm{p}$

Smith DM (1985) The Practice of Silviculture, 8th ed. John Wiley \& Sons, New York, NY, ÉtatsUnis, $527 \mathrm{p}$

Stiell WM, Berry AB (1973) Yield of unthinned red pine plantations at the Petawawa Forest Experiment Station. Department of the Environment, Canadian Forestry Service, Publication No 1320, $16 \mathrm{p}$

Stiell WM, Berry AB (1977) A 20-year-old trial of red pine planted at seven spacings. Department of the Environment of Canada, Canadian Forestry 
Service, Forest Management Institute, Information Report FMR-X-97, $25 \mathrm{p}$

Tessier L, Nola P, Serre-Bachet F (1994) Deciduous Quercus in the Mediterranean region: tree-ring/climate relationships. New Phytol 126, 355-367

Van Deusen PC (1990) Stand dynamics and red spruce decline. Can J For Res 20, 743-749

Van Deusen PC, Reams, GA (1993) Frequency domain tree-ring standardization. For Sci 39, 5565

Vose JM, Swank WT (1994) Effects of long-term drought on the hydrology and growth of a white pine plantation in the southern Appalachians. For Ecol Manage 64, 25-39

Whitmore FW, Zahner R (1966) Development of the xylem ring in stems of young red pine trees. For Sci $12,198-210$
Wijk S (1986) Influence of climate and age on annual shoot increment in Salix herbacea. J Ecol 74, 685-692

Wilkes $\mathbf{J}$ (1989) Variation in wood density of Pinus radiata in New South Wales, Australia. Can J For Res 19, 289-294

Yin X, Foster NW, Morrison IK, Arp PA (1994) Treering-based growth analysis for a sugar maple stand : relations to local climate and transient soil properties. Can J For Res 24, 1567-1574

Zahner R, Stage AR (1966) A procedure for calculating daily moisture stress and its utility in regressions of tree growth on weather. Ecology 47, 64-74

Zahner R, Lotan JE, Baughman WD (1964) Earlywoodlatewood features of red pine grown under simulated drought and irrigation. For Sci 10, 361-370

Zimmermann MH, Brown CL (1971) Trees: Structure and Function. Springer-Verlag, New York, NY, États-Unis 